\title{
Seizures in horses: diagnosis and classification
}

This article was published in the following Dove Press journal:

Veterinary Medicine: Research and Reports

5 October 2015

Number of times this article has been viewed

\section{Véronique A Lacombe}

Department of Physiological Sciences, Oklahoma State University Center for Veterinary Health Sciences, Stillwater, OK, USA
Correspondence: Véronique A Lacombe Department of Physiological Sciences, Oklahoma State University Center for Veterinary Health Sciences, 283 McElroy Hall, Stillwater, OK 74078, USA

Tel +l 4057448089

Fax + I 4057448263

Email veronique.lacombe@okstate.edu
Abstract: Seizures are a diverse and very common set of chronic neurologic disorders in humans and dogs but are less common in horses. Seizures refer to a specific clinical event (described as sudden and severe) regardless of the etiology, which includes both intracranial and extracranial causes. Therefore, after briefly reviewing some definitions, this article aims to describe the use of a standardized classification, which could facilitate a logical approach for the clinician to establish a diagnosis, as well as to use a consistent mode of communication. For instance, seizures can be classified by type (ie, focal vs generalized) or etiology (ie, reactive, symptomatic, cryptogenic, idiopathic). In particular, epilepsy, a brain disorder characterized by recurrent seizures can be classified as primary (ie, genetic origin) or secondary (ie, acquired). This review further discusses the limitations associated with the clinical workup of horses with seizures. This is germane to the fact that the identification of the underlying cause remains challenging due to the technical limitations of imaging the equine adult brain. Indeed, as in man and dogs, epilepsies of unknown cause (ie, cryptogenic) account for the majority of all epilepsies. Therefore, although electroencephalography and advanced brain imaging techniques (eg, computed tomography and magnetic resonance imaging) are becoming increasingly available, information obtained from the history, physical, and neurologic examinations and progression of clinical signs and response to treatment remain essential in the workup of horses with seizures.

Keywords: focal seizure, generalized seizure, symptomatic, cryptogenic, electroencephalography, computed tomography

\section{Introduction}

Seizure disorders are common chronic neurological conditions in humans and dogs, with an estimated prevalence of $0.8 \%-0.1 \%$ and $0.5 \%-5.7 \%$, respectively. ${ }^{1,2}$ Seizures, which are characterized by a sudden, often catastrophic event, also affect many veterinary species but are less common in horses because of a relatively high seizure threshold. Therefore, both clinical and experimental studies on seizures in this species are rather limited. Epileptic seizures are clinical manifestations of rapid, excessive, or hypersynchronous abnormal neuronal activity from the cerebral cortex. ${ }^{3}$ Therefore, epileptic seizures are signs of cerebral dysfunction caused by an imbalance between excitation and inhibition in the brain. Thus, a true epileptic seizure has a specific neural origin and can occur as a single or recurrent event. Epilepsy (from the Greek word epilambanein, meaning to be seized or attacked) is a brain disorder characterized by an enduring predisposition to generate recurrent $(\geq 2)$ epileptic seizures. ${ }^{3}$ 


\section{Classification}

The diagnosis of seizures in horses is complicated by the diversity of terms used by equine clinicians. For instance, seizure-like disorders are also referred to as fits, attacks, strokes, convulsions, grand mal, petit mal, or epilepsy. Therefore, a common terminology for seizure-like disorders in horses is needed to establish a consistent mode of communication among clinicians and to help them in the establishment of a diagnosis. ${ }^{4}$ In humans, the commission on classification and terminology of the International League Against Epilepsy (ILAE) first created a comprehensive classification for epilepsy and epileptic syndromes $\sim 30$ years ago. ${ }^{3,5-7}$ This ILAE nomenclature is based on a diagnostic scheme organized in five axes that includes phenomenology (defined as the description of the event; axis 1), type of seizures (axis 2), the epilepsy syndrome (characterized by a cluster of specific symptoms; axis 3), etiology of seizures (axis 4), and axis 5, which is an optional designation of the degree of impairment caused by the epileptic condition. ${ }^{8,9}$ This classification is a dynamic process as it is updated on a regular basis. ${ }^{10-12}$ Although there are over 40 epileptic syndromes reported in humans, ${ }^{2}$ axis 3 has been rarely used in veterinary medicine. Although a systemic and comprehensive classification of seizures (mainly using axes 2 and 4) has been established in dogs and cats, ${ }^{13-17}$ similar classification has only been recently reported in a large (>100 cases) referral equine-based population. ${ }^{4,9}$ In these studies, seizures were classified according to revised consensus of the ILAE and based on epileptological studies in small animals. ${ }^{8,13}$

\section{Ictal phenomenology (axis I)}

It consists of the description of the horse's behavior and clinical signs during the "pre-ictal", "post-ictal", and "interictal" periods, which are characteristic of epileptic seizures. Therefore, careful observation of these phases could help the clinician to differentiate epileptic from nonepileptic seizures. Just before the seizure (aura), horses may exhibit signs of anxiety and uneasiness. The ictal phase is characterized by the loss of control (in various degrees), the paroxystic nature of the attacks (ie, they start suddenly and terminate suddenly), and the repetitive clinical pattern (ie, attacks are identical from episode to episode). ${ }^{18}$ For instance, during the episode of a generalized seizure, horses may become unconscious and have symmetric clonic muscle contractions, followed by symmetric tonic muscle contractions. Horses may also show deviation of eyeballs, dilated pupils, ptyalism, trismus or jaw clamping, opisthotonos, lordosis or kyphosis, violent paddling movements of the limbs, uncontrolled urination and defecation, and excessive sweating. A generalized seizure may last from 5 seconds to a few minutes. Status epilepticus is defined as more than 30 minutes of continuous seizure activity or more sequential seizures with no complete recovery of consciousness between seizures. It is uncommon in adult horses. ${ }^{19}$ After the seizure (postictus), horses may show depression, stupor and/or blindness for hours to days. For instance, following a generalized seizure, the horse regains its consciousness but may remain disorientated, uncoordinated, and seems exhausted. ${ }^{20}$

\section{Classification by type (axis 2 )}

Seizures have a wide range of clinical manifestations, which may range from mild alterations in consciousness, focal muscle fasciculations to recumbency with tonic-clonic struggling. The first clinical signs of seizures are usually highly indicative of the region of the cerebral cortex involved. Therefore, seizures can be further classified by type (ie, focal vs generalized seizures; Figure 1). Generalized seizures involve the entire cerebral cortex and result in generalized bilateral motor activity over the whole body, including convulsive seizures (formerly referred to "grand-mal") or nonconvulsive seizures (formerly referred to "petit-mal") or myoclonic seizures. ${ }^{9}$ Consciousness may be impaired, and this impairment may be the initial manifestation. Generalized seizures can originate from both cerebral hemispheres from the onset (primary generalized seizures) or progress from focal seizure (secondary generalized seizures). ${ }^{9}$ In the later case, the epileptic foci that originate from a localized region of the peripheral cerebral cortex rapidly project to the entire brain. Focal (also referred to as partial) seizures, which are caused by a localized abnormal discharge mostly from the periphery of the cerebrum, result in localized motor signs or sensations, mostly asymmetrical, which may be indicative of

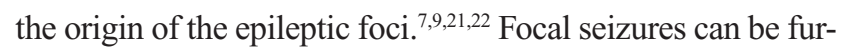
ther categorized as simple, if alertness and normal mentation are maintained; or complex, if impairment of consciousness is reported. ${ }^{9}$ Tremors/fasciculations involving the facial muscle, abnormal tonus/clonus in one limb, or paroxysmal behavior changes (including repetitive manic biting at the ground, selfmutilation, or violent headshaking) could be suggestive of focal seizures. Horses may exhibit both focal and generalized seizures. Therefore, it might be difficult for the clinician to differentiate between primary vs secondary generalized seizures when a focal seizure rapidly progresses to generalized seizure. ${ }^{9,13}$ In addition, focal seizures may also be in the form of subtle and/or transient clinical signs that may go undetected or be interpreted as the pre-ictal phase of an impending seizure. ${ }^{9}$ Therefore, 


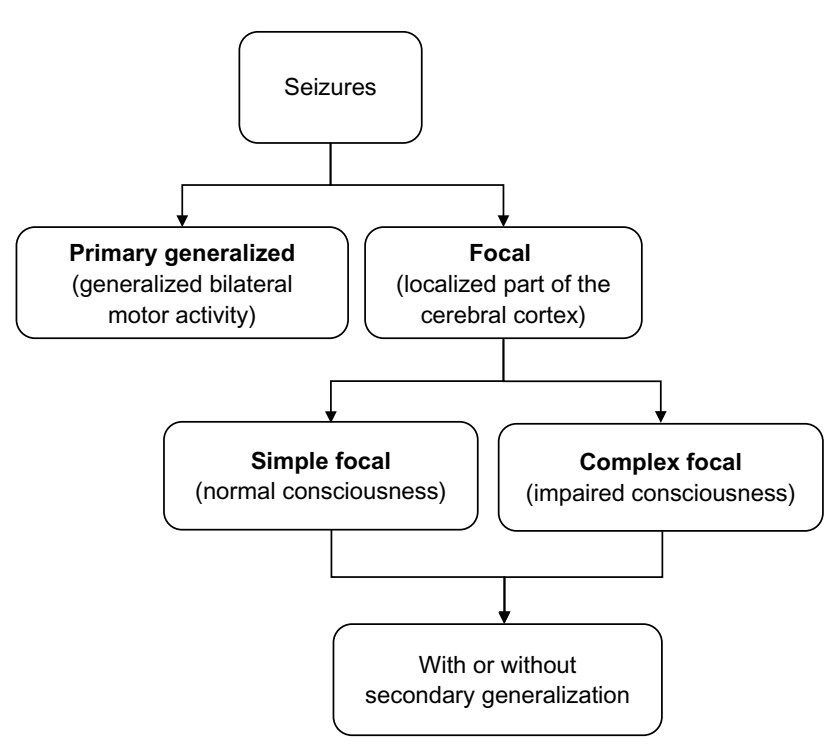

Figure I Classification of seizures by type in horses.

clinical observation of the onset of seizures may allow to better identifying and classifying focal seizures in horses, as for other species. ${ }^{9,23}$ It has been reported that most common types of seizures observed in an equine referral center were complex focal seizures with or without secondary generalization, which is in agreement with data in small animals (Figure 1). 13,17,24 Complex focal seizures are commonly observed in neonatal foals and are referred to as "chewing-gum fits", jaw chomping, and lip smacking. ${ }^{25}$

\section{Classification by etiology (axis 4)}

Seizures can also be classified by etiology (ie, reactive, symptomatic, cryptogenic, idiopathic, Figure 2). Reactive seizures are occurring from the reaction of a healthy brain to a temporary systemic disease that includes electrolyte abnormalities (eg, hyponatremia, hypocalcemia), hepatic disease, uremia, septicemia, or toxin exposure. ${ }^{26}$ Therefore, reactive seizures are not considered true epilepsy since the patient is not affected by a brain disorder. ${ }^{17}$ Epilepsy is classified as primary (ie, genetic origin) or secondary (ie, acquired). Secondary epilepsy further includes symptomatic and cryptogenic. Epilepsy is classified as symptomatic if a structural brain disorder (eg, inflammatory, neoplastic, or traumatic) is identified on the basis of abnormal results of neurodiagnostic testing. In a recent retrospective study in horses, symptomatic epilepsies were identified in $36 \%$ of cases because of identifiable underlying chronic structural brain disorders, including intracranial neoplasia (eg, lymphoma, oligodendrioma, pituitary adenoma), cholesterol granuloma, vascular events, meningitis, and head trauma. ${ }^{4}$ However, the lack of abnormalities on neurodiagnostic testing does not exclude the possibility of intracranial lesions. Therefore, when intracranial origin of seizures is suspected despite unremarkable results of neurodiagnostic tests, recurrent seizures are classified as cryptogenic epilepsy (Greek cryptos $=$ hidden), also referred to as probable symptomatic epilepsy. For instance, magnetic resonance imaging (MRI)

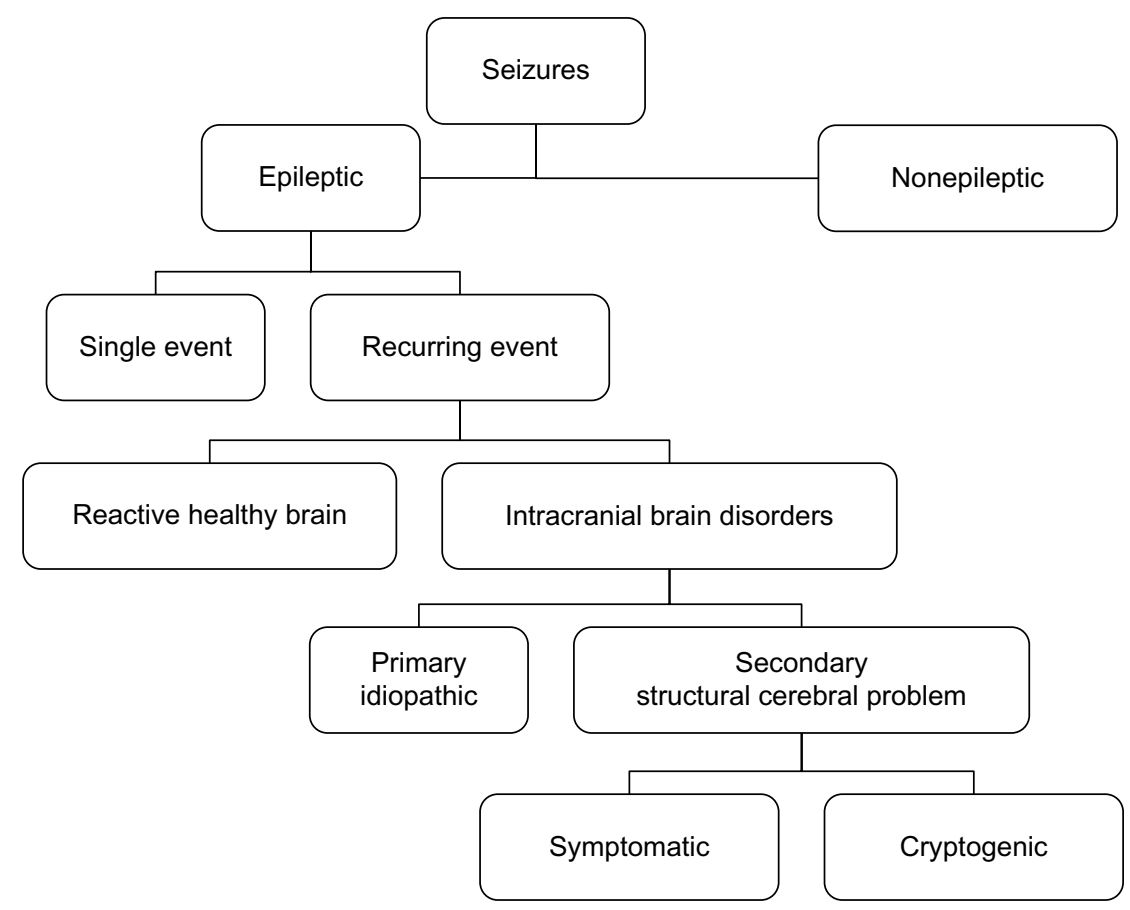

Figure 2 Etiologic classification of seizures in horses. 
of the skull was normal in 29 of 84 horses with epilepsy. ${ }^{27} \mathrm{In}$ addition, cryptogenic epilepsy was reported in $55 \%$ of horses affected by seizure-like disorders. ${ }^{4}$ Therefore, as in man and dogs, epilepsies of unknown cause (ie, cryptogenic) account for the majority of all epilepsies, ${ }^{12,28}$ demonstrating the challenges associated with the diagnosis of seizures. ${ }^{4}$ Idiopathic epilepsy refers to epilepsy with a presumed genetic origin and without an identifiable structural cause. In humans and dogs, the timing of idiopathic epilepsy is fairly well known, allowing patients with a specific pattern of histories and signalments to be assigned to this category. ${ }^{1,28}$ Indeed, the estimated prevalence of primary (ie, idiopathic) epilepsy in dogs is high, ranging from approximately $0.5 \%$ to $5.7 \%{ }^{1}$ In contrast, idiopathic epilepsy appears to be rare in horses with the exception of Arabian foals with juvenile idiopathic epilepsy. ${ }^{4,29,30}$ Although one should not confuse idiopathic (ie, "primary") with cryptogenic (ie, "secondary") epilepsy, the distinction can be rather arbitrary. ${ }^{28}$ Therefore, the ILAE commission recently reclassified idiopathic epilepsy as "genetic" epilepsy and cryptogenic epilepsy as epilepsy of "unknown cause", underlying the fact that establishment of such classification is a dynamic process. ${ }^{12}$

\section{Diagnosis}

The diagnosis of seizures remains challenging because seizures refer to a specific clinical event regardless of the underlying cause, which can be difficult to determine due to the technical limitations of imaging the equine adult brain. In addition, the etiology of seizures is diverse and includes both intracranial (eg, neoplasia, head trauma, cerebral abscess, vascular events, encephalitis or meningitis, hypoxic-ischemic encephalopathy) and extracranial diseases (ie, hepatic encephalopathy, electrolyte imbalances, toxic insult), as previously described. ${ }^{19}$ Therefore, a complete diagnostic evaluation is required to determine the cause of seizures and to classify seizures by etiology, although it may expensive and time consuming. ${ }^{31}$

\section{History and physical examination}

Since the clinician rarely observes the seizure episode, a thorough historical investigation is required and may allow the identification of potential precipitating factors (eg, noise, lightning strike, intracranial injection, traumatic event). If it can be performed safely, video surveillance could be used to record the episodes and to monitor the horse's behavior and sleep patterns. In addition, since there is a high risk of trauma during the ictal phase and since many horses are unobserved for long periods of the day, the presence of a seizure might be solely suspected after recognition of unexplained trauma to the head or legs and/or the presence of corneal ulcer. History and physical examination should also rule out noncerebral conditions that mimic seizure activity, such as tetanus, botulism, and hyperkalemic periodic paralysis (HYPP), as well as painful conditions, such as colic, limb fractures, and exertional myopathy. In these conditions, horses do not lose consciousness but remain bright and alert. Attempts should also be made to differentiate epileptic seizures from collapse of cardiovascular origin (eg, cardiac arrhythmias or heart failure) and narcolepsy/cataplexy. ${ }^{32}$ For instance, the clinician should perform an electrocardiogram and/or a 24-hour Holter recording to identify arrhythmia. In addition, narcoleptic horses that collapse may show rapid eye movement sleep. One should perform a complete neurologic examination during the interictal period, since an immediate postictal examination may reveal depression, weakness, blindness, and crossed extensor reflex, which may lead to false anatomic localization of lesions. ${ }^{19}$ If the seizures are caused by a structural cerebral problem, cranial deficit could be observed along with weakness and/or ataxia of the limbs. However, normal neurological exams do not rule out epileptic seizures since neurological examinations can be completely normal between episodes. Furthermore, any sedatives or anticonvulsants given to control the seizures will affect subsequent neurological examination.

\section{Blood and cerebrospinal fluid analysis}

A complete serum biochemistry profile should be performed to rule out metabolic abnormalities that may trigger seizures (ie, reactive). Analysis of complete blood count and fibrinogen could indicate inflammation (leukocytosis and hyperfibrinogenemia) or viral infection. Tests for bile acids and ammonia should be performed if hepatic encephalopathy is suspected. Presence of hypoglycemia may indicate a cause for seizures, although the likelihood of hypoglycemia-induced seizures is rare. ${ }^{33}$ Renal disease and hyperlipidemia should be ruled out as well. Hyperkalemia is the hallmark of HYPP, which may be confused with seizure-like activity. Cerebrospinal fluid (CSF) analysis is a valuable tool in determining the underlying etiology of seizures caused by an active structural cerebral abnormality (ie, symptomatic), in which neutrophilic pleiocytosis, lymphocytic pleiocytosis, or albuminocytologic dissociation may be observed. However, normal CSF analysis can be seen in many cases. Xanthochromia suggests an increased protein in the CSF, although prior hemorrhage may result in xanthochromia. In addition, it might be difficult to differentiate whether an increase in total protein count reflects the underlying cause or is 
the consequence of the seizure episode itself. ${ }^{4}$ Neutrophilia is suggestive of viral encephalitidies (eg, togavirus such as Eastern equine encephalitis, Venezuelan equine encephalitis, Western equine encephalitis), and serologic testing of serum and CSF is then warranted. ${ }^{19}$ Testing for equine protozoal myeloencephalitis should also be considered since seizures have been reported in this population. ${ }^{34}$ Culture of the CSF is indicated if there is any suggestion of sepsis. ${ }^{19}$

\section{Electroencephalography}

Electroencephalography (EEG) is a relatively simple and noninvasive neurophysiological technique of determining abnormal cerebral electrical activity. ${ }^{35}$ Brain waves are recorded by placing electrodes on the scalp (eg, frontal, occipital, parietal areas) and using different montages (eg, reference or bipolar circular). The difference of potential detected between pairs of electrodes or between an electrode and its reference is amplified, filtered, and then recorded. ${ }^{35-37}$ Similar to humans, sleep waves (eg, beta and theta waves) are detected on the EEG recording of horses with the exception of alpha waves. ${ }^{20}$ In addition, the ictal EEG reflects bilateral neuronal discharge widespread in both hemispheres during generalized seizures. Therefore, EEG is a standard diagnostic procedure for the investigation and management of human patients with epilepsy, in particular in establishing probability of recurrence, need for treatment, and prognosis..$^{37,38}$ In horses, EEG was initially used to study natural sleep and its disorders by implanting surgically electrodes in the skull and to monitor anesthetic depth during general anesthesia. ${ }^{39-46}$ This diagnostic test has also been used as a screening procedure in horses with seizure disorders because of its excellent sensitivity, similar to reports in dogs. ${ }^{36,38}$ For instance, EEG recording revealed a generalized abnormal electrical activity before the development of any clinical signs in horses with experimental West-Nile virus encephalitis. ${ }^{47}$ In addition, EEG has been shown to be useful for the diagnosis of seizures associated with intracranial disorders in this species, similar to reports in small animals. ${ }^{36,48}$ However, there are few studies reporting the use of EEG in evaluating neurological diseases in horses, and there is little agreement on the use of a standardized protocol. ${ }^{20}$ Additional limitations of this electrodiagnostic test are related to the facts that 1) only electrical activity arising from the superficial part of the cerebral cortex is recorded; 2) the establishment of normal values remains difficult because frequency and amplitudes are state dependant (awake, drowsy, sleeping, sedated, anesthetized), varies with age (foal vs adult), and chemical restraint influences EEG patterns; 3) the lack of recording of epileptical activity does not automatically rule out seizures;
4) it requires extensive expertise from the clinician to interpret the reading; and 5) it is mostly limited to some large private practices and neurological referral institutions. ${ }^{35}$

EEG can be performed on awake or sedated animals or on animals under general anesthesia. The main advantage of performing EEG on sedated or anesthetized horses is that it will significantly reduce artifacts caused by head, ear, and eye movements of the patient, as well as by auditory and visual stimuli. For example, eye movements result in large loop-like signals. ${ }^{20,49}$ However, in humans and small animals, recordings on awake patients are optimal because the use of sedation (eg, xylazine, ketamine) and a fortiori, the use of general anesthesia (eg, halothane, isoflurane), influence EEG patterns by altering cortical activity. ${ }^{38,44,50}$ Therefore, cautious interpretation of the EEG recording obtained under such condition is crucial to avoid overinterpretation of druginduced changes in EEG background. ${ }^{35}$ Finally, the use of tranquilizers may increase the threshold for seizures, thus reducing or masking potential epileptiform activity on EEG recording. Interestingly, ketamine appears to have both proconvulsant and anticonvulsant properties in humans because of its potential antagonist action on $\mathrm{N}$-methyl-D-aspartate receptor. ${ }^{51}$ Therefore, one should consider repeating EEG examination in a sedated or anesthetized animal suspected of having seizures and for which the first EEG recording was normal. ${ }^{19,35}$ In humans, frequent and prolonged sophisticated EEG monitoring is routinely performed, and ambulatory EEG, which does not require sedation, has been recently shown to be suitable as a diagnostic tool in horses. The main advantage of this technique is to potentially increase the likelihood of recording abnormalities since it allows long-lasting recordings (eg, up to 48 hours) while the horse is able to display normal daily activity and sleep patterns. ${ }^{20,52}$

Accurate interpretation of the EEG recording can provide useful information in regard to the presence of cerebral disease. One should assess the background of the EEG recording for abnormal frequency and amplitude, the presence of asymmetrical patterns between regions, and the presence of paroxysmal activity. ${ }^{19,35,36,53}$ Normal EEG background has been determined in anesthetized adult horses (frequency of 8-13 Hz and a voltage of $25-50 \mu \mathrm{V}$ ). In neonatal foals, the normal patterns include high-voltage-slow wave activity. ${ }^{36,54}$ The most common EEG abnormalities associated with cerebral diseases are the change in either amplitude or frequency. ${ }^{35}$ Increase toward low voltage-fast activity or high voltage-slow activity has been observed in epileptic patients and is suggestive of ongoing irritative processes or neuronal death, respectively. ${ }^{35,36,55}$ Focal, multifocal, or generalized 
epileptiform activity can be recorded on EEGs of epileptic patients, depending on the localization of the discharges and the extent of the cerebral cortex involved. ${ }^{29,36,53,56}$ For instance, generalized EEG changes may reflect diffuse cortical or subcortical disease such as infection, trauma, idiopathic epilepsy, as well as systemic metabolic disease (eg, hepatic encephalopathy, hypocalcemia). ${ }^{35,36}$ For instance, acute cases of equine encephalitis were characterized by periodic episodes of generalized high voltage activity. ${ }^{47,57}$ Focal abnormal electrical activity may reflect focal cortical disease (eg, hemorrhage, tumor, or abscessation). However, similar to other species, it has been reported that the EEG pattern of horses with intracranial masses, such as abscesses, includes generalized continuous high voltage slow wave activity and is not specific as to localization. ${ }^{36,58,59}$ Therefore, abnormal EEG recording is not pathognomonic of a disease but rather reflect the kind of process occurring (eg, acute vs chronic; focal vs diffuse; inflammatory vs degenerative). ${ }^{35}$ Importantly, the presence of epileptiform paroxysmal activity (either generalized or focal) on the EEG recording supports the diagnosis of seizures and helps to differentiate between epileptic and other nonepileptic paroxysmal attacks. ${ }^{16,35,60}$ Indeed, we recently reported paroxysmal transient events (eg, wave, spike and wave, or polyspike and wave complexes) on the majority of EEG recordings of horses with epilepsy. ${ }^{4}$

\section{Advanced imaging diagnostic techniques}

Skull radiographs may help to determine the presence of traumatic skull fracture (eg, fracture of the basi-sphenoid bone) or the presence abscess/tumor in the sinus. However, small skull fractures may remain undetected by conventional radiographs, due to superimpositions of bony structures. Therefore, MRI and/or computed tomography (CT) should be used to detect any structural abnormalities in the brain and the skull (eg, tumor, hemorrhage, hydrocephalus, fractures). Similar to conventional X-ray imaging, CT of the head measures the attenuation of X-ray photon beams through different tissues, except that radiation passes successively through tissues from multiple directions. ${ }^{61-63}$ Since the densest structure of the head is bone on CT imaging, this technique has superior capabilities for imaging bony structures. Although it allows visualizing the anatomy of bony structures of the head in great details, small fractures may be missed if appropriate window parameters are not chosen. ${ }^{61}$ Thus, CT should be primarily conducted in cases of seizures secondary to head trauma and could be warranted to rule out structural cerebral lesions. ${ }^{24}$ However, with the exception of subarachnoid hemorrhage and mineralized lesions, CT showed limited sensitivity in identifying inflammatory disorders and small soft tissue lesions in the equine brain. ${ }^{61}$ In addition, we recently reported that CT imaging of the head was normal in $69 \%$ of horses presented for seizure-like disorders for which an abnormal EEG tracing was recorded. ${ }^{4}$ In addition, although the use of intravenous iodinated contrast agent could identify alteration of the blood-brain barrier or vascular abnormalities, the use of contrast did not help further in identifying cerebral lesions in horses presented for neurologic deficits. ${ }^{24}$ However, standing $\mathrm{CT}$ remains an attractive option for the equine clinician since it limits the risks and cost associated with the use of general anesthesia in horses with neurological deficits. ${ }^{64,65}$ In general, MRI is superior to CT for the evaluation of physiological and pathophysiological processes of the central nervous system because of the better contrast that can be achieved between soft tissues, by exploiting the properties of hydrogen within tissue water and lipids. ${ }^{61,64,66}$ Thus, MRI is more sensitive than CT in detecting CNS lesions such as demyelinating and other white matter diseases, cerebral neoplasms, degenerative diseases, and nonacute hemorrhage. ${ }^{61,62}$ Therefore, MRI has become the gold standard test to diagnose structural intracranial abnormalities in small animals. In addition, a positive neuroanatomical localization was identified between MRI and postmortem findings in horses with equine head disorders. ${ }^{27}$ Unfortunately, most referral centers do not have MR imaging equipment to accommodate an equine adult head. In addition, recent studies demonstrated the lack of sensitivity of MRI in diagnosing seizures in both horses and dogs, reinforcing the need of EEG to establish diagnosis and prognosis in epileptic patients. ${ }^{23,27}$ Indeed, a 2007 evidence-based review from the American Academy of Neurology and the American Epilepsy Society recommends an electroencephalogram combined with a CT or an MRI scan in the workup of seizures in humans. ${ }^{67}$

\section{Conclusion}

The diagnosis of seizures remains a challenge in equine medicine. Not only results from the diagnostic tests but also information obtained from the history, physical, and neurologic examinations and progression of clinical signs and response to treatment remain essential in the diagnostic workup of horses with seizures. In addition, there is a need for common agreement on the terminology use in equine epileptology. Therefore, a standardized classification could facilitate a logical approach for the clinician to establish a diagnosis, a therapeutic plan, and a prognosis for seizures.

\section{Acknowledgment}

We would like to thank Tim Vojt for the medical illustration. 


\section{Disclosure}

The author reports no conflicts of interest in this work.

\section{References}

1. Ghormley TM, Feldman DG, Cook JR Jr. Epilepsy in dogs five years of age and older: 99 cases (2006-2011). J Am Vet Med Assoc. 2015;246(4):447-450.

2. Chandler K. Canine epilepsy: what can we learn from human seizure disorders? Vet J. 2006;172(2):207-217.

3. Fisher RS, Boas WV, Blume W, et al. Epileptic seizures and epilepsy: definitions proposed by the International League against Epilepsy (ILAE) and the International Bureau for Epilepsy (IBE). Epilepsia. 2005;46(4):470-472.

4. Lacombe VA, Mayes M, Mosseri S, Reed SM, Fenner WR, Ou HT. Epilepsy in horses: aetiological classification and predictive factors. Equine Vet J. 2012;44(6):646-651.

5. Beghi E, Berg A, Carpio A, et al. Comment on epileptic seizures and epilepsy: definitions proposed by the International League Against Epilepsy (ILAE) and the International Bureau for Epilepsy (IBE) Epilepsia. 2005;46(10):1698-1699.

6. Commission on Classification and Terminology of the International League Against Epilepsy. Proposal for revised classification of epilepsies and epileptic syndromes. Epilepsia. 1989;30(4):389-399.

7. Commission on Epidemiology and Prognosis, International League Against Epilepsy. Guidelines for epidemiologic studies on epilepsy. Epilepsia. 1993;34(4):592-596.

8. Engel J. A proposed diagnostic scheme for people with epileptic seizures and with epilepsy. Report of the ILAE task force on classification and terminology. Epilepsia. 2001;42(6):796-803.

9. Lacombe VA, Mayes M, Mosseri S, Reed SM, Ou TH. Distribution and predictive factors of seizure types in 104 cases. Equine Vet $J$. 2014;46(4):441-445.

10. Ferrie $\mathrm{CD}$. Terminology and organization of seizures and epilepsies: radical changes not justified by new evidence. Epilepsia. 2010;51(4):713-714.

11. Avanzini G. A sound conceptual framework for an epilepsy classification is still lacking. Epilepsia. 2010;51(4):720-722.

12. Berg AT, Berkovic SF, Brodie M, et al. Revised terminology and concepts for organization of seizures and epilepsies: report of the ILAE commission on classification and terminology, 2005-2009. Epilepsia. 2010;51(4):676-685.

13. Berendt M, Gram L. Epilepsy and seizure classification in 63 dogs: a reappraisal of veterinary epilepsy terminology. J Vet Intern Med. 1999; 13(1):14-20.

14. Podell M, Fenner WR, Powers JD. Seizure classification in dogs from a nonreferral-based population. J Am Vet Med Assoc. 1995;206(11): $1721-1728$.

15. Schriefl S, Steinberg TA, Matiasek K, Ossig A, Fenske N, Fischer A. Etiologic classification of seizures, signalment, clinical signs, and outcome in cats with seizure disorders: 91 cases (2000-2004). $J \mathrm{Am}$ Vet Med Assoc. 2008;233(10):1591-1597.

16. Podell M. Seizures in dogs. Vet Clin North Am Small Anim Pract. 1996;26(4):779-809.

17. Pakozdy A, Halasz P, Klang A. Epilepsy in cats: theory and practice. J Vet Intern Med. 2014;28(2):255-263.

18. Berendt M. Epilepsy. In: Braund KG, editor. Clinical Neurology in Small Animals - Localization, Diagnosis and Treatment. Ithaca, New York, USA: International Veterinary Information Service; 2004.

19. Lacombe VA. Seizures, narcolepsy and cataplexy. In: Reed SM, Bayly W, Sellon D, editors. Equine Internal Medicine. St Louis, MO: Saunders; 2010:572-578.

20. van der Ree M, Wijnberg I. A review on epilepsy in the horse and the potential of ambulatory EEG as a diagnostic tool. Vet Q. 2012;32(3-4): 159-167.

21. Berendt M, Gredal H, Alving J. Characteristics and phenomenology of epileptic partial seizures in dogs: similarities with human seizure semiology. Epilepsy Res. 2004;61(1-3):167-173.
22. Berendt M, Gredal H, Ersboll AK, Alving J. Premature death, risk factors, and life patterns in dogs with epilepsy. JVet Intern Med. 2007;21(4):754-759.

23. Viitmaa R, Cizinauskas S, Bergamasco LA, et al. Magnetic resonance imaging findings in Finnish spitz dogs with focal epilepsy. J Vet Intern Med. 2006;20(2):305-310.

24. Sogaro-Robinson C, Lacombe VA, Reed SM, Balkrishnan R. Factors predictive of abnormal results for computed tomography of the head in horses affected by neurologic disorders: 57 cases (2001-2007). J Am Vet Med Assoc. 2009;235(2):176-183.

25. Furr M. Perinatal asphyxia in foals. Compend Conint Educ Pract. 1996;18(12):1342-1351.

26. Hardefeldt LY. Hyponatraemic encephalopathy in azotaemic neonatal foals: four cases. Aust Vet J. 2014;92(12):488-491.

27. Manso-Diaz G, Dyson SJ, Dennis R, et al. Magnetic resonance imaging characteristics of equine head disorders: 84 cases (2000-2013). Vet Radiol Ultrasound. 2015;56(2):176-187.

28. Shorvon SD. The etiologic classification of epilepsy. Epilepsia. 2011;52(6):1052-1057.

29. Aleman M, Gray LC, Williams DC, et al. Juvenile idiopathic epilepsy in Egyptian Arabian foals: 22 cases (1985-2005). J Vet Intern Med. 2006;20(6):1443-1449.

30. Lichter-Peled A, Polani S, Stanyon R, Rocchi M, Bar-Gal GK. Role of KCNQ2 and KCNQ3 genes in juvenile idiopathic epilepsy in Arabian foals. Vet J. 2013;196(1):57-63.

31. Kube SA, Aleman M, Williams DC, LeCouteur RA. How to work up a horse with seizures. Paper presented at: 50th Annual Convention of the American Association of Equine Practitioners 2004; Denver, Colorado.

32. Lyle CH, Turley G, Blissitt KJ, et al. Retrospective evaluation of episodic collapse in the horse in a referred population: 25 cases (1995-2009). JVet Intern Med. 2010;24(6):1498-1502.

33. Wong D, Hepworth K, Yaeger M, Miles K, Wilgenbusch C. Imaging diagnosis-hypoglycemia associated with cholangiocarcinoma and peritoneal carcinomatosis in a horse. Vet Radiol Ultrasoun. 2015;56(1): E9-E12.

34. Dunigan CE, Oglesbee MJ, Podell M, Mitten LA, Reed SM. Seizures activity associated with equine protozoal myeloencephalitis. Prog Vet Neurol. 1995;6(2):50-54.

35. Lacombe V, Andrews M. Electrodiagnostic evaluation of the nervous system. In: Reed S, Furr M, editors. Equine Neurology. Ames, IA: Blackwell publishing Co; 2007:127-148.

36. Lacombe VA, Podell M, Furr M, et al. Diagnostic validity of electroencephalography in equine intracranial disorders. JVet Intern Med. 2001; 15(4):385-393.

37. Aminoff M. Electroencephalography: general principles and clinical applications. In: Aminoff M, editor. Electrodiagnosis in Clinical Neurology. 2nd ed. New York, NY: Churchill Livingstone; 1986:21-75.

38. Redding RW. Electroencephalography. In: Oliver JE, Horlein BF, Mayhew IG, editors. Veterinary Neurology. Philadelphia, PA: WB Saunders; 1987:111-145.

39. Grabow JD, Anslow RO, Spalatin J. Electroencephalographic recordings with multicontact depth probes in a horse. Am J Vet Res. 1969;30(7): 1239-1243.

40. Garner HE, Geddes LA, Amend JF, Rosborou Jp, Ross JN. Electrodes for recording cortical electroencephalograms in ponies. Lab Anim Sci. 1972;22(2):262-265.

41. Otto K, Short CE. Electroencephalographic power spectrum analysis as a monitor of anesthetic depth in horses. Vet Surg. 1991;20(5):362-371.

42. Murrell JC, Johnson CB, White KL, Taylor PM, Haberham ZL, WatermanPearson AE. Changes in the EEG during castration in horses and ponies anaesthetized with halothane. Vet Anaesth Analg. 2003;30(3):138-146.

43. Johnson CB, Taylor PM. Comparison of the effects of halothane, isoflurane and methoxyflurane on the electroencephalogram of the horse. Brit J Anaesth. 1998;81(5):748-753.

44. Auer JA, Amend JF, Garner HE, Hutcheson DP, Salem CA. Electroencephalographic responses during volatile anesthesia in domestic ponies - comparative-study of isoflurane, enflurane, methoxyflurane, and halothane. J Equine Med Surg. 1979;3(3):130-134. 
45. Haga HA, Dolvik NI. Electroencephalographic and cardiovascular variables as nociceptive indicators in isoflurane-anaesthetized horses. Vet Anaesth Analg. 2005;32(3):128-135.

46. Dallaire A, Ruckebus Y. Sleep and Wakefulness in housed pony under different dietary conditions. Can J Comp Med. 1974;38(1):65-71.

47. Lapras M, Florio R, Joubert L. Normal electroencephalogram in the horse. Its pathological variations in West Nile viral meningoencephalomyelitis. Relations to clinical findings, virology and histopathology. Rev Med Vet (Toulouse). 1968;119:673-693.

48. Knecht CD, Sorjonen DC, Simpson ST. Ancillary tests in the diagnosis of seizures. J Am Anim Hosp Assoc. 1984;20(3):455-458.

49. Williams DC, Aleman M, Tharp B, et al. Qualitative and quantitative characteristics of the electroencephalogram in normal horses after sedation. J Vet Intern Med. 2012;26(3):645-653.

50. Purohit RC, Mysinger PW, Redding RW. Effects of xylazine and ketamine-hydrochloride on the electroencephalogram and the electrocardiogram in the horse. Am J Vet Res. 1981;42(4):615-619.

51. Borris DJ, Bertram EH, Kapur J. Ketamine controls prolonged status epilepticus. Epilepsy Res. 2000;42(2-3):117-122.

52. Wijnberg ID, van der Ree M, van Someren P. The applicability of ambulatory electroencephalography (AEEG) in healthy horses and horses with abnormal behaviour or clinical signs of epilepsy. Vet Quart. 2013;33(3):121-131.

53. Lacombe V. Seizure disorders. In: Robinson N, Sprayberry K, editors. Current Therapy in Equine Medicine. 7th ed. St Louis, MI: Saunders; 2015:375-379.

54. Mysinger PW, Redding RW, Vaughan JT, Purohit RC, Holladay JA. Electroencephalographic patterns of clinically normal, sedated, and tranquilized newborn foals and adult horses. Am J Vet Res. 1985;46(1):36-41.

55. Itabisashi T, Horino R, Hirano K, Maeda M. Electroencephalographic observation on sheep and cattle with experimental cerebrocortical necrosis. Jpn J Vet Sci. 1990;52(3):551-558.
56. Berendt M, Hogenhaven H, Flagstad A, Dam M. Electroencephalography in dogs with epilepsy: similarities between human and canine findings. Acta Neurol Scand. 1999;99(5):276-283.

57. Heath SE, Artsob H, Bell RJ, Harland RJ. Equine encephalitis caused by snowshoe hare (California Serogroup) Virus. Can Vet J. 1989;30(8):669-671.

58. Hepburn RJ, Furr MO. Sinonasal adenocarcinoma causing central nervous system disease in a horse. J Vet Intern Med. 2004;18(1):125-131.

59. Steiss JE, Cox NR, Knecht CD. Electroencephalographic and histopathologic correlations in eight dogs with intracranial mass lesions. Am J Vet Res. 1990;51(8):1286-1291.

60. Noachtar S, Remi J. The role of EEG in epilepsy: a critical review. Epilepsy Behav. 2009;15(1):22-33.

61. Lacombe VA, Sogaro-Robinson C, Reed SM. Diagnostic utility of computed tomography imaging in equine intracranial conditions. Equine Vet J. 2010;42(5):393-399.

62. Gilman S. Medical progress - Imaging the brain - Second of two parts. New Engl J Med. 1998;338(13):889-896.

63. Lacombe VA. Challenges associated with the diagnosis and treatment of parasitic encephalitis. Equine Vet Educ. 2013;25(12):605-606.

64. Jose-Cunilleras E, Piercy RJ. Advanced diagnostic imaging options in horses with neurological disease that localises to the head. Equine Vet Educ. 2007;19(4):179-181.

65. Porter EG, Werpy NM. New concepts in standing advanced diagnostic equine imaging. Vet Clin North Am Equine Pract. 2014;30(1):239-268.

66. Tucker RL, Farrell E. Computed tomography and magnetic resonance imaging of the equine head. Vet Clin North Am Equine Pract. 2001; 17(1):131-144.

67. Krumholz A, Wiebe S, Gronseth G, et al. Practice parameter: Evaluating an apparent unprovoked first seizure in adults (an evidence-based review) - Report of the quality standards subcommittee of the American Academy of Neurology and the American Epilepsy Society. Neurology. 2007;69(21):1996-2007.
Veterinary Medicine: Research and Reports

\section{Publish your work in this journal}

Veterinary Medicine: Research and Reports is an international, peer-reviewed, open access journal publishing original research, case reports, editorials, reviews and commentaries on all areas of veterinary medicine. The manuscript management system is completely online and includes a very quick and fair peer-review system.

\section{Dovepress}

Visit http://www.dovepress.com/testimonials.php to read real quotes from published authors. 